\title{
Robust Prediction of Atrial Fibrillation Termination Using Wavelet Bidomain Entropy Analysis
}

\author{
R Alcaraz ${ }^{1}$, JJ Rieta $^{2}$ \\ ${ }^{1}$ Innovation in Bioengineering Research Group. University of Castilla-La Mancha, Cuenca, Spain \\ ${ }^{2}$ Biomedical Synergy. Valencia University of Technology, Valencia, Spain
}

\begin{abstract}
The ability to predict if an AF episode terminates spontaneously or not is a challenging clinical problem. This work presents a robust AF prediction method carried out by estimating, through regularity indexes, the atrial activity (AA) organization increase prior to AF termination. This regularity variation appears as a consequence of the decrease in the number of reentries into the atrial tissue. $A A$ was obtained from surface ECG recordings using an average QRST template cancellation technique. Wavelet transform (WT) was used in a bidomain way (time and frequency) in order to improve organization estimation. Thereafter, a more robust classification process for terminating and non-terminating AF episodes was developed making use of two different wavelet decomposition strategies. Finally, the AA organization both in time and wavelet domains was estimated. Trougth the application of this strategy, 96\% of the terminating and non-terminating analyzed AF episodes were correctly classified.
\end{abstract}

\section{Introduction}

The treatment of Atrial Fibrillation (AF) patients is still unsatisfactory, which can be attributed to the progressing nature of this arrhythmia and its physiological mechanisms provoking that the onset and the termination of $\mathrm{AF}$ episodes are still unexplained [1]. Moreover, AF episodes that terminate spontaneously (paroxysmal AF episodes) are the preceding stage of permanent $\mathrm{AF}$ episodes, which only terminate applying pharmacological, electrical or surgical intervention. Permanent AF patients have a high embolic accidents risk and about $18 \%$ of paroxysmal AF degenerate into permanent AF in less than 4 years [1]. Therefore, the early prediction of AF maintenance is crucial, because appropriate interventions may terminate the arrhythmia and prevent $\mathrm{AF}$ chronification. In contrast, the spontaneous AF termination prediction could avoid unnecessary therapy, and consequently, reduce the associated clinical costs and improve the patient's quality of life.
It has been shown that the decrease in the number of reentries prior to $\mathrm{AF}$ termination produces simpler wavefronts into the atrial tissue [2], and $f$ waves, that characterize the ECG in AF, evolve to $P$ waves, which characterize the normal sinus rhythm [3]. Therefore, the atrial activity (AA) becomes more organized before AF termination [4] and, as a consequence, this fact can be used to predict AF termination when the proper analysis tools are used.

To reduce noise, ventricular residues and any other nuisance signal in the AA, a new methodology based on wavelet transform (WT) and bidomain sample entropy (SampEn) is presented in this work. The WT allows to isolate certain time frequency characteristics of a signal in limited decomposition coefficients. This fact permits to observe regularity variations in the AA signal, that would be left masked in other cases [5]. SampEn was used to estimate the AA organization in a bidomain way (time and frequency). This regularity estimator was selected because electrical remodelling in AF is a far-from-linear process [6]. This phenomenon is described as the progressive shortening of effective atrial refractory periods, thus increasing the number of simultaneous reentries and, as a consequence, the perpetuation of $\mathrm{AF}$ [1].

\section{Materials}

Fifty electrocardiogram (ECG) Holter recordings of one minute and two leads (II and V1) available in Physionet [7] were analyzed. The database included non-terminating AF episodes (group N), which were observed to continue in $\mathrm{AF}$ for, at least, one hour following the end of the excerpt, and AF episodes terminating immediately after the end of the extracted segment (group T). Recordings were divided into a learning and a test set. Next, 10 labelled recordings of each group composed the learning set. An optimal threshold, which should allow to discern $\mathrm{N}$ from $\mathrm{T}$ episodes, was defined making use of the proposed methodology applied to the learning set. Finally, the test set was composed with the remaining 30 recordings.

The ECG recordings were preprocessed in order to reduce noise, nuisance interferences and improve later anal- 
ysis. Firstly, baseline wander was removed making use of bidirectional high pass filtering with $0.5 \mathrm{~Hz}$ cutt-off frequency [8]. Secondly, high frequency noise was reduced with a eight order bidirectional IIR Chebyshev low pass filtering, whose cut-off frequency was $70 \mathrm{~Hz}$ [9]. Finally, powerline interference was removed through adaptive filtering, which preserves the ECG spectral information [10].

\section{Methods}

\subsection{Wavelet transform (WT)}

The Continuous Wavelet Tranform (CWT) is defined as the sum over all time of the signal $f(t)$ scaled with a factor $a$ and shifted a certain time interval $b$ through the mother wavelet function $\Psi$ [11]:

$$
\begin{gathered}
C(a, b)=\int_{-\infty}^{\infty} f(t) \cdot \Psi_{a, b}(t) d t \\
\Psi_{a, b}(t)=\frac{1}{\sqrt{a}} \Psi\left(\frac{t-b}{a}\right) \quad \forall a, b \in \Re^{+}
\end{gathered}
$$

The results of the CWT are many wavelet coefficients $C$, which are a function of scale and position. Concretely, a wavelet coefficients vector is obtained for each analyzed scale [11]. Discrete time Wavelet Transform (DWT) is the sampled version of the CWT in a dyadic grid. One advantage of the DWT is that the original signal can be synthesized using fewer coefficients than in the case of the CWT. An efficient way to implement the DWT using filters was developed by Mallat, and was called multiresolution algorithm [12].

\subsection{Sample entropy}

Sample Entropy (SampEn) examines time series for similar epochs and assigns a non-negative number to the sequence, with larger values corresponding to more complexity or irregularity in the data [13]. Two input parameters, a run length $m$ and a tolerance window $r$, must be specified for SampEn to be computed. $\operatorname{SampEn}(m, r)$ is the negative logarithm of the conditional probability that two sequences similar during $m$ points remain similar at the next point, where self-matches are not included in calculating the probability. A detailed mathematic description can be found in [13].

Although $m$ and $r$ are critical in determining the outcome of SampEn, no guidelines exist for optimizing their values. Nevertheless, the $m$ and $r$ values suggested by Pincus are $m=1$ or $m=2$ and $r$ between 0.1 and 0.25 times the standard deviation of the original time serie [14].

\subsection{Proposed classification methodology}

In this classification strategy (see Fig. 1), cancellation of QRST waves from the preprocessed ECG signals was performed. Though a variety of QRST cancellation techniques exist $[15,16]$, ventricular cancellation in AF recordings is a difficult task which is strongly dependent on the number of available leads. Therefore, the average QRST template cancellation method was used, since only two leads were available [17]. Next, the power spectral density (PSD) of the residual signal was calculated using Welch Periodogram. A Hamming window of 4096 points in length, a 50\% overlapping between adjacent windowed sections and a 8192-points Fast Fourier Transform (FFT) were used as computational parameters. The frequency with the largest amplitude within the $3-9 \mathrm{~Hz}$ range was selected as the dominant atrial frequency $\left(f_{p}\right)$. Later, a AA wavelet decomposition was performed and two alternatives for processing the obtained wavelet coefficients vectors were proposed in order to reduce ventricular residues and noise, which are provoked by differences between average QRST complex and each individual beat.

In block 1 (see Fig. 1), the wavelet coefficients vector, and consequently the frequency band containing the dominant atrial frequency, was reconstructed back to the timedomain. SampEn of this time reconstructed signal was calculated. This procedure was introduced in previous works with promising results [5]. In block 2 , the same vector was linearly interpolated. Hence, a wavelet coefficients vector with equal number of samples than the original signal was obtained. Finally, the interpolated vector regularity was estimated using SampEn.

The results obtained through the independent application of each block to the learning set defined the optimum SampEn thresholds (Th1 and Th2) that, later, will allow the test set classification into terminating and non-terminating AF episodes.

The block 2 strategy was proposed to improve the classification into terminating and non-terminating AF episodes obtained through block 1 [5]. With the block 1, nonterminating AF signals that presented a high $f_{p}$ were incorrectly classified, whereas with the block 2 , terminating AF signals that have a small $f_{p}$ were also incorrectly discriminated, as will be presented in next section. Thereby, the classification process was improved by combining both blocks as follows:

- if an AF episode was differently classified through both blocks, and:

- $f_{p}$ was lower than a threshold of $f_{p}(\mathrm{Th})$, that was obtained using the learning set, the classification obtained with block 1 was considered.

- $f_{p}$ was higher than $\mathrm{Th}$, the classification performed with block 2 was chosen.

- On the contrary, the result of both blocks was coincident and any of them can be selected indistinguishably.

In our experiments, the best results were obtained with a 7 level decomposition and "bior4.4" as wavelet family. 


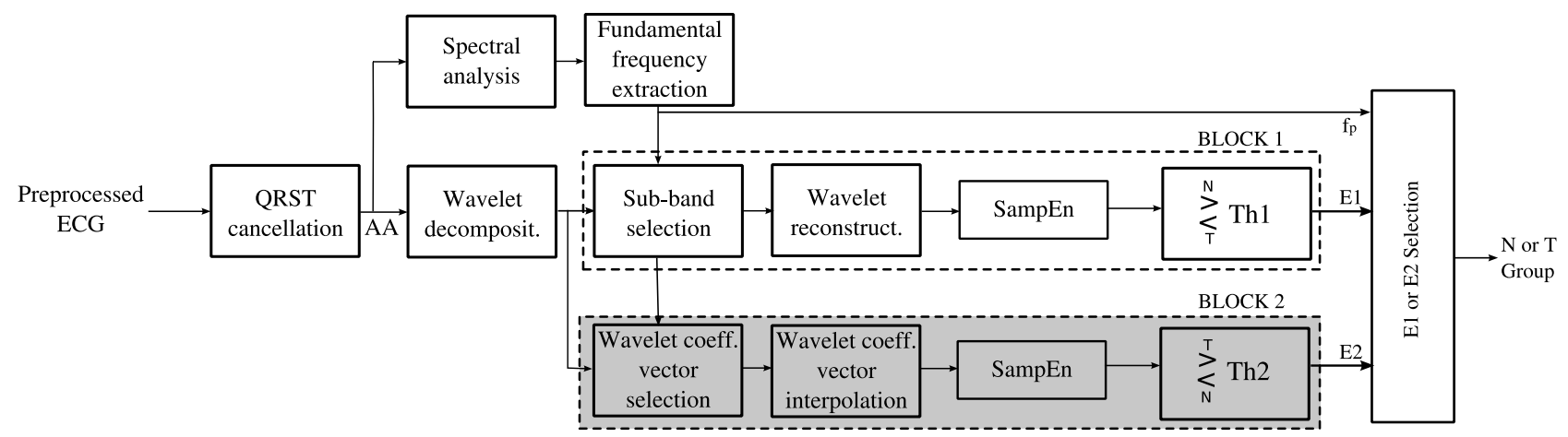

Figure 1. Block diagram describing the proposed classification methodology.

\section{Results}

Each block was independently applied to the learning set. With block $1,80 \%$ sensitivity and $100 \%$ specificity were obtained, see Fig. 2(a). The Receiver Operating Characteristic (ROC) curve provided 0.0902 as optimun SampEn discrimination threshold between terminating and non-terminating AF episodes. Fig 2(b) shows the SampEn values for the 20 learning signals together with the mean and standard deviation values for each group. Note that all terminating and 8 out of 10 non-terminating recordings were correctly discriminated. The non-terminating episodes $f_{p}$ that were incorrectly classified was 6.25 and $7.35 \mathrm{~Hz}$ respectively.

With block 2, 100\% sensitivity and 70\% specificity were obtained, see Fig 2(a). The ROC curve provided 0.034 as optimun SampEn discrimination threshold. All nonterminating and 7 out of 10 terminating $\mathrm{AF}$ episodes were correctly classified, such as Fig. 2(c) shows. The values 4, 4.75 and $4.75 \mathrm{~Hz}$ were the three terminating dominant atrial frequencies of the incorrectly discriminated signals. In this case, $5.5 \mathrm{~Hz}$ was chosen as the dominant atrial frequency threshold (Th).

By applying the combined block1-block2 strategy to the learning signals, all non-terminating and terminating AF episodes were correctly classified. Using the aforementioned SampEn and dominant atrial frequency thresholds, 28 out of 30 test signals were correctly discerned. Therefore, the AF behavior of 48 out of 50 recordings (96\%) were correctly predicted through the AA organization analysis into the time and wavelet domains.

\section{Discussion and conclusions}

The results obtained with block 1 show that terminating episodes present lower SampEn values and, consequently, higher signal organization than non-terminating ones. This observation agrees with the organization increase in the AA prior to AF termination reported with invasive atrial electrograms [18] that, at this moment, is clinically accepted [4].

Regarding block 2 , for a specific scale, the wavelet coefficients vector contains the temporal evolution of the similarity between analyzed signal and scaled mother wavelet. A high regularity value of this time series indicates that the analyzed signal presents a constant waveform during the studied time period. On the contrary, a low regularity value implies a variable waveform. Because of the AA evolution from $f$ waves to $\mathrm{P}$ waves that is produced in $\mathrm{AF}$ recordings prior to its termination [3], the results obtained with block 2 can be justified. These results show that terminating episodes present a more irregular wavelet coefficients vector associated to the AA main component than non-terminating episodes. Thus, the structural organization produced in $\mathrm{AF}$ surface recordings, because of the decrease in the number of reentries before $\mathrm{AF}$ termination, can be used to predict AF termination when the proper analysis tools are used.

The organization analysis with SampEn of the AA main component obtained with WT (i. e. time reconstruction of the frequency band containing the dominant atrial frequency) was presented in [5], and $90 \%$ of terminating and non-terminating AF episodes were correctly classified. This work demonstrates that a more robust methodology can be obtained by adding the wavelet domain regularity analysis of the AA main component (i. e. the wavelet coefficients interpolated vector), because $96 \%$ of the AF episodes were correctly discriminated.

\section{Acknowledgements}

This work was partly supported by the projects 20070086 from the R+D+i Vice-rectorate of the Valencia University of Technology, GV06/299 from Consellería de Empresa, Universidad y Ciencia de la Generalitat Valenciana and TEC2007-64884 from the Spanish Ministry of Education and Science. 


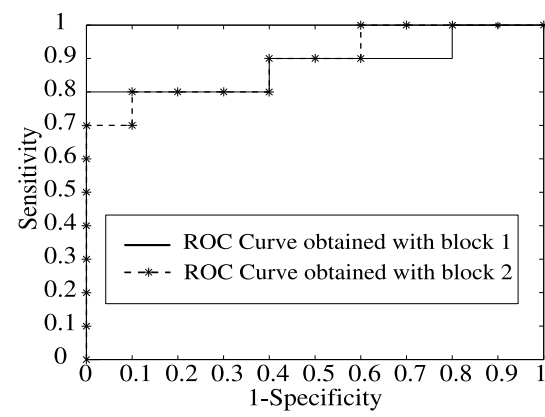

(a)

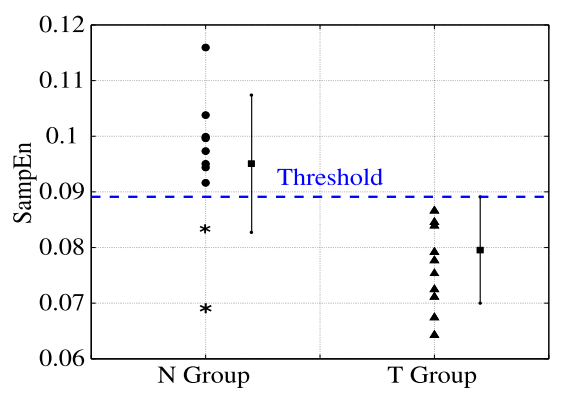

(b)

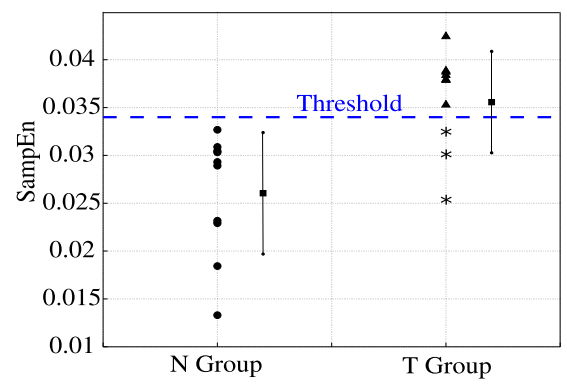

(c)

Figure 2. Obtained results. (a) Receiver Operating Characteristic (ROC) curve obtained with block 1 and 2 applied to the learning set. Classification into terminating and non-terminating AF episodes obtained with (b) block 1 and (c) block 2.

\section{References}

[1] Fuster V, Ryden LE, Asinger RW, et al. ACC/AHA/ESC 2006 guidelines for the management of patients with atrial fibrillation: a report of the American College of Cardiology/American Heart Association Task Force on practice guidelines and the European Society of Cardiology committee for practice guidelines developed in collaboration with th european heart rhythm association and the heart rhythm society. Europace 2006;8(9):651-745.

[2] Konings K, Kirchhof C, Smeets J, et. al. High-density mapping of electrically induced atrial fibrillation in humans. Circulation 1994;89:1665-1680.

[3] Blomstrom-Lundqvist C, Scheinman MM, Aliot EM, Alpert JS, et. al. ACC/AHA/ESC guidelines for the management of patients with supraventricular arrhythmias. European Heart Journal 2003;24(20):1857-1897.

[4] Bollmann A, Lombardi F. Electrocardiology of atrial fibrillation - Current knowledge and future challenges. IEEE Engineering in Medicine and Biology Magazine 2006; 25(6):15-23.

[5] Alcaraz R, Vayá C, Cervigón R, Sánchez C, Rieta JJ. Wavelet sample entropy: A new approach to predict termination of atrial fibrillation. The IEEE Inc Computers in Cardiology 2006;33:597-600.

[6] Bollmann A. Quantification of electrical remodeling in human atrial fibrillation. Cardiovasc Res 2000;47:207-209.

[7] Goldberger AL, Amaral LA, Glass L, et. al. Physiobank, physiotoolkit, and physionet: components of a new research resource for complex physiologic signals. Circulation 2000; 101(23):e215-e220.

[8] Dotsinsky I, Stoyanov T. Optimization of bi-directional digital filtering for drift suppression in electrocardiogram signals. J Med Eng Technol 2004;28(4):178-180.

[9] Sun Y, Chan K, Krishnan SM. ECG signal conditioning by morphological filtering. Comput Biol Med 2002; 32(6):465-479.

[10] Ferdjallah M, Barr RE. Adaptive digital notch filter design on the unit-circle for the removal of powerline noise from biomedical signals. IEEE Transaction on Biomedical Engineering 1994;42(6):529-536.

[11] Addison PS. The Illustrated Wavelet Transform Handbook. Introductory Theory and Applications in Science, Engineering, Medicine and Finance. Institute of Physics Publishing, 2002. ISBN 0-7503-0692-0.

[12] Mallat SG. A theory for multiresolution signal decomposition: The wavelet representation. IEEE Trans Pattern Anal Mach Intell 1989;11(7):674-693. ISSN 0162-8828.

[13] Richman JS, Moorman JR. Physiological time series analysis using approximate entropy and sample entropy. Am J Physiol 2000;278(6):H2039-H2049.

[14] Pincus SM. Approximate entropy as a measure of system complexity. Proc Natl Acad Sci USA 1991;88(6):22972301.

[15] Rieta JJ, Castells F, Sánchez C, Zarzoso V, Millet J. Atrial activity extraction for atrial fibrillation analysis using blind source separation. IEEE Trans Biomed Eng 2004; 51(7):1176-1186.

[16] Stridh M, Sörnmo L. Spatiotemporal QRST cancellation techniques for analysis of atrial fibrillation. IEEE Trans Biomed Eng 2001;48(1):105-111.

[17] Slocum J, Sahakian A, Swiryn S. Diagnosis of atrial fibrillation from surface electrocardiograms based on computerdetected atrial activity. J Elec 1992;25(1):1-8.

[18] Takanashi Y, Sanders P, Jaïs P, Hocini M, et. al. Organization of frequency spectra of atrial fibrillation: Relevance to radiofrequency catheter ablation. Journal of Cardiovascular Electrophysiology 2006;17(4):382-388.

Address for correspondence:

Raúl Alcaraz Martínez

E. U. Politécnica de Cuenca

Campus Universitario

16071 Cuenca (Spain)

raul.alcaraz@uclm.es 